\title{
Development of Attentional Control of Verbal Auditory Perception From Middle to Late Childhood: Comparisons to Healthy Aging
}

\author{
Susanne Passow \\ Max Planck Institute for Human Development, Berlin, Germany, \\ and University of Bergen
}

\author{
René Westerhausen and Kenneth Hugdahl \\ University of Bergen and Haukeland University Hospital
}

\author{
Hauke R. Heekeren \\ Max Planck Institute for Human Development, Berlin, Germany, \\ and Freie Universität Berlin
}

\author{
Maike Müller \\ Central Institute of Mental Health, Heidelberg University
}

Isabell Wartenburger

University of Potsdam

Ulman Lindenberger

Max Planck Institute for Human Development, Berlin, Germany

Shu-Chen Li

Max Planck Institute for Human Development, Berlin, Germany, and Technische Universität Dresden

\begin{abstract}
Multitalker situations confront listeners with a plethora of competing auditory inputs, and hence require selective attention to relevant information, especially when the perceptual saliency of distracting inputs is high. This study augmented the classical forced-attention dichotic listening paradigm by adding an interaural intensity manipulation to investigate developmental differences in the interplay between perceptual saliency and attentional control during auditory processing between early and middle childhood. We found that older children were able to flexibly focus on instructed auditory inputs from either the right or the left ear, overcoming the effects of perceptual saliency. In contrast, younger children implemented their attentional focus less efficiently. Direct comparisons of the present data with data from a recently published study of younger and older adults from our group suggest that younger children and older adults show similar levels of performance. Critically, follow-up comparisons revealed that younger children's performance restrictions reflect difficulties in attentional control only, whereas older adults' performance deficits also reflect an exaggerated reliance on perceptual saliency. We conclude that auditory attentional control improves considerably from middle to late childhood and that auditory attention deficits in healthy aging cannot be reduced to a simple reversal of child developmental improvements.
\end{abstract}

Keywords: child development, attentional control, auditory perception, aging, dichotic listening

In everyday life, children, just like other listeners, are often confronted with situations in which they need to focus on the speech stream of one particular individual (e.g., teacher, parent, or classmate), whereas others are talking at the same time in the background. In such situations, a plethora of different sound sources competes for the brain's limited processing resources, and
This article was published Online First December 31, 2012.

Susanne Passow, Center for Lifespan Psychology, Max Planck Institute for Human Development, Berlin, Germany, and Department of Biological and Medical Psychology, University of Bergen, Bergen, Norway; Maike Müller, Department of Cognitive and Clinical Neuroscience, Central Institute of Mental Health, Medical Faculty Mannheim, Heidelberg University, Mannheim, Germany; René Westerhausen Department of Biological and Medical Psychology, University of Bergen, Bergen, Norway, and Division of Psychiatry, Haukeland University Hospital, Bergen, Norway; Kenneth Hugdahl, Department of Biological and Medical Psychology, University of Bergen, and Division of Psychiatry and Department of Radiology, Haukeland University Hospital; Isabell Wartenburger, Center for Excellence Cognitive Sciences, Department of Linguistics, University of Potsdam, Potsdam, Germany; Hauke R. Heekeren, Center for Lifespan Psychology, Max Planck Institute for $\mathrm{Hu}-$ man Development, and Department of Education and Psychology, Freie Uni- versität Berlin, Berlin, Germany; Ulman Lindenberger, Center for Lifespan Psychology, Max Planck Institute for Human Development; Shu-Chen Li, Center for Lifespan Psychology, Max Planck Institute for Human Development, and Department of Psychology, Technische Universität Dresden, Dresden, Germany.

This study was funded through the Max Planck Society and a doctorate fellowship granted to Susanne Passow by the Max Planck International Research Network on Aging (MaxNetAging). Special thanks go to the student assistants for their valuable support during data collection and to all children who participated in the study and their parents.

Correspondence concerning this article should be addressed to Susanne Passow, Department of Biological and Medical Psychology, University of Bergen, Jonas Lies vei 91, N-5009 Bergen, Norway. E-mail: susanne .passow@psybp.uib.no 
children have to selectively attend, for instance, to the teacher's voice (relevant information) and inhibit other voices (irrelevant information). According to the biased competition model of attention, the demand for attentional control dynamically varies with the perceptual saliency of relevant information (Desimone \& Duncan, 1995; Miller \& Cohen, 2001). For instance, the demand to selectively focus on the teacher would be low when the auditory inputs received from the teacher are perceptually more salient than other competing auditory inputs coming from the classroom environment, and vice versa. How perceptual saliency may interact with attentional control during auditory perception and how this interaction may differ between middle and late childhood has yet not been investigated and was, thus, the main aim of the present study. Moreover, no study has directly contrasted this interaction during child development with that during aging. Therefore, a second aim of the study was to extend the adult development literature by directly comparing and contrasting the current child developmental data with results from an earlier study on healthy aging (Passow et al., 2012) to gain a more comprehensive understanding of attentional control of auditory perception across the life span.

\section{Development of Auditory Perception and Attentional Control During Childhood}

Relative to higher order cognitive mechanisms, the human auditory system matures rapidly. Basic aspects of auditory processing, such as frequency, intensity, and duration discrimination already function well 6 months after birth (e.g., Trehub, Bull, \& Thorpe, 1984; Trehub, Thorpe, \& Morrongiello, 1987) and reach adultlike levels in early childhood (e.g., Stalinski, Schellenberg, \& Trehub, 2008; Werner, 1996). Hearing sensitivity continuously improves from infancy to early childhood and reaches adultlike levels at school age (Trehub, Schneider, Morrongiello, \& Thorpe, 1988). The precision of segregating different sound patterns, however, is still greater in adults than in children, reflecting that the maturation of complex auditory pattern analysis takes longer compared with the maturation of simpler discrimination processes (Sussman, Wong, Horvath, Winkler, \& Wang, 2007).

Although basic auditory discrimination matures in early childhood, children have to learn to selectively attend to relevant information and inhibit distracting irrelevant information in situations in which multiple sensory inputs compete for further processing. Younger children in the range of 5-12 years are more susceptible to distractions from irrelevant information across a broad range of auditory and visual tasks than adolescents and young adults (e.g., Gomes, Molholm, Christodoulou, Ritter, \& Cowan, 2000; Ridderinkhof, van der Molen, Band, \& Bashore, 1997; Rueda et al., 2004; Waszak, Li, \& Hommel, 2010). For instance, in the visual domain, the Flanker task (Eriksen \& Eriksen, 1974) is commonly used to investigate developmental changes in attentional control. Flankers (distractors) that are incongruent with the target elicit greater interference effects and demand for more attentional control than congruent flankers. From early to late childhood (e.g., from 5 to 11 years as reported in Ridderinkhof et al., 1997), these effects of interfering flankers decrease (Li, Hämmerer, Müller, Hommel, \& Lindenberger, 2009; Rueda et al., 2004; Waszak et al., 2010).
In the auditory domain, younger children have more difficulties in selectively attending to specific auditory information and in ignoring irrelevant information than older children (see Gomes et al., 2000, for a review). Dichotic listening tasks are often used to investigate developmental differences in auditory attentional control. In this task, two different auditory inputs are simultaneously presented to the right and left ear, and the participants are asked to either attend to both ears (neutral-focus condition) and report the syllable they heard most clearly or they are instructed to focus their attention on one ear and report only the stimuli presented to this ear (i.e., the focused-right or focused-left condition). Dichotic listening performance is typically expressed in laterality indices, reflecting the percentage of right-ear reports in relation to left-ear reports. The laterality index ranges from -100 to $+100 \%$ (Marshall, Caplan, \& Holmes, 1975). A positive laterality index indicates a right-ear advantage (REA), that is, more correct reports of right-ear syllables, whereas a negative laterality index indicates a left-ear advantage (LEA) with more correct reports from the left ear. In the neutral-focus condition, if verbal auditory inputs (e.g., consonant-vowel [CV] syllables) are used, adult participants typically show a REA reflecting the left-hemispheric lateralization for speech processing (for a review, see Hugdahl, 2003; Kimura, 1967). Relative to the neutral-focus condition, the REA is typically enhanced when attention is focused on the right ear and decreased or even reversed to an LEA when attention is focused on the left ear. The relative shifts in the extent of the REA as a function of attentional focus are thought to reflect the flexibility of attentional control (see Hugdahl, 2003, for a review). In the neutral-focus condition, a reliable REA (i.e., a left-hemispheric superiority for speech processing) can already be observed in children aged 5 years (e.g., Berlin, Lowe-Bell, Hughes, \& Berlin, 1973; Harper \& Kraft, 1994; Westerhausen, Helland, Ofte, \& Hugdahl, 2010). In the focused-attention conditions in contrast to older children (e.g., aged 10-11 years), younger children are not able to flexibly exert their attentional control, and the REA is, thus, much less modulated as a function of attentional focus (e.g., Hugdahl, Carlsson, \& Eichele, 2001; Takio et al., 2009).

Extending the classical dichotic listening task, recent studies showed that an intensity-modulated dichotic listening task combined with attentional manipulation is particularly amenable for investigating the interactions between perceptual saliency and attentional control during auditory processing (Passow et al., 2012; Tallus, Hugdahl, Alho, Medvedev, \& Hamalainen, 2007; Westerhausen et al., 2009). In this variant of the dichotic listening paradigm, the relative perceptual saliency of the two dichotically presented syllables and the attentional focus are manipulated simultaneously. The perceptual saliency is varied by gradually changing the degree of intensity differences between the ears, favoring either the right or left ear (see the top portion of Figure $1 \mathrm{~A}$ for a schematic illustration). As in the classical dichotic listening task, attentional focus is varied by instructing the participants to attend to both ears or to focus on either the right or the left ear (see the bottom portion of Figure 1A for a schematic illustration). The laterality indices change as a function of the interaction between perceptual saliency and attentional focus. Relative to the neutral-focus conditions, participants with intact attentional control typically report more syllables from the right ear in focusedright conditions and more syllables from the left ear in focused-left conditions. This is reflected in increased laterality indices in 
A

\section{Perceptual Saliency Manipulation}

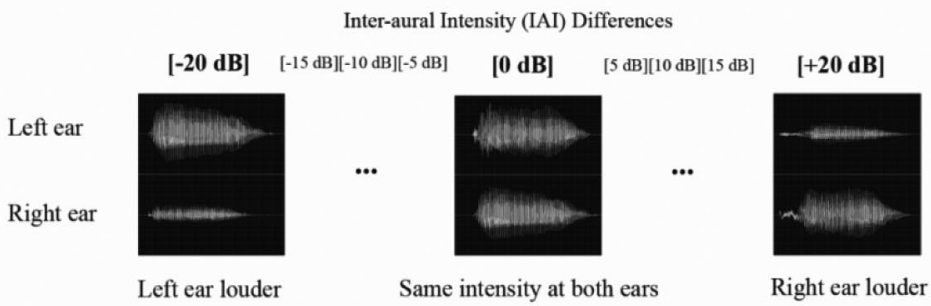

Attentional Focus Manipulation
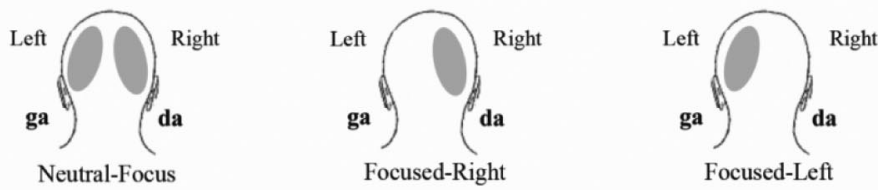

B Schematic Illustration of Expected Modulation of the Laterality Index

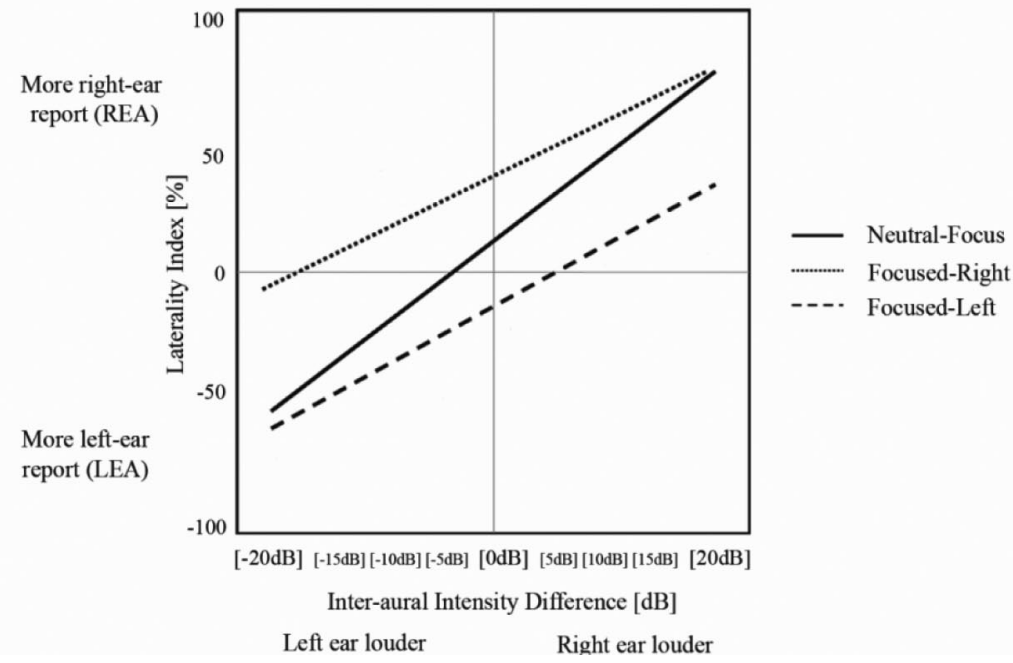

Figure 1. Schematic illustrations of (A) the perceptual saliency and attentional focus manipulation and (B) the expected modulation of the laterality index.

focused-right and decreased laterality indices in focused-left conditions (see Figure 1B for a schematic illustration).

Previous studies showed that younger adults are able to use attentional control to manipulate the laterality indices throughout all levels of perceptual saliency. Specifically, the laterality index in the neutral-focus conditions was, overall, lower compared with the laterality index in the focused-right conditions, but higher compared with the laterality index in the focused-left conditions (Passow et al., 2012; Tallus et al., 2007; Westerhausen et al., 2009). Furthermore, the magnitude of the differences between neutralfocus and the focused-attention conditions was larger when attentional focus and perceptual saliency favor different ears compared with when attentional focus and perceptual saliency favor the same ear. In comparison to younger adults, older adults were not able to direct their attentional focus flexibly, and their performance was dominated by perceptual saliency (Passow et al., 2012).
To the best of our knowledge, no other study as yet has investigated the development of the interaction between perceptual saliency and attentional focus from middle to late childhood. In life span samples covering the age range from early childhood to old age, various cognitive functions such as conflict resolution (Waszak et al., 2010), inhibitory mechanisms (Dempster, 1992), episodic memory (Shing \& Lindenberger, 2011), or perceptual speed in the visual domain (Kail \& Salthouse, 1994) follow an inverted U-shaped function of rise and fall, reflecting that both children and older adults perform below the level of younger adults. However, direct comparisons revealed that this apparent behavioral symmetry does not necessarily point to an identity of the mechanisms that are operating in the course of child development and healthy aging (e.g., Baltes, Lindenberger, \& Staudinger, 2006; Craik \& Bialystok, 2006; Hämmerer, Li, Müller, \& Lindenberger, 2011; Li et al., 2004, 2009; Shing \& Lindenberger, 2011; 
Waszak, Schneider, Li, \& Hommel, 2009). Thus far, little is known about differences or similarities in the underlying mechanisms of attentional control of auditory perception between child development and healthy aging. Older adults' deficits in auditory processing relative to younger adults likely reflect effects of compromised sensory functions that are further augmented by agingrelated attentional deficits (e.g., Helfer \& Freyman, 2008; Passow et al., 2012; Tun, O'Kane, \& Wingfield, 2002). In contrast, agerelated improvements in dichotic listening performance from middle to late childhood are likely to primarily or exclusively reflect age-graded changes in attentional control (Hugdahl et al., 2001; Ridderinkhof et al., 1997; Rueda et al., 2004; Takio et al., 2009; Waszak et al., 2010), given that basic auditory mechanisms reach adultlike levels in early childhood (e.g., Stalinski et al., 2008; Trehub et al., 1988; Werner, 1996).

\section{Study Aims and Hypotheses}

In this study, we focused on the development of the interaction between perceptual saliency and attentional control during auditory processing from middle to late childhood. To this end, we compared a group of children aged 7-8 years with a group of children aged 11-12 years using an intensity-modulated dichotic listening task that included an attentional manipulation. On the basis of studies reporting an increase in attentional control during childhood (e.g., Takio et al., 2009; Waszak et al., 2010), we expected that older relative to younger children would modulate their attentional control more flexibly to overcome irrelevant inputs from the nonattended ear. Hence, we hypothesized that attending to the right ear would increase the laterality index across all perceptual saliency conditions relative to the neutral-focus condition, whereas attending to the left ear would reduce the laterality index. In line with previous studies in adult samples (Passow et al., 2012; Tallus et al., 2007; Westerhausen et al., 2009), we expected that the differences between the laterality indices in the neutral-focus and focused-attention conditions in older children would be magnified when perceptual saliency and attentional focus favor different ears as opposed to conditions favoring the same ear. In contrast, we expected that the laterality indices in younger children would be less affected by the attentional manipulation because their relatively less mature attentional system would not be strong enough to counteract salient but attention-incongruent auditory input. Accordingly, we predicted that younger children would show smaller differences between the laterality indices in neutral-focus and focused-attention conditions than older children. To explore differences between maturational and senescent influences on the interaction between perceptual saliency and attentional control during auditory processing, we also conducted secondary analyses in which we compared selected results of the present study with corresponding data from a previously published study with younger and older adults (Passow et al., 2012).

\section{Method}

\section{Participants}

The child development sample consisted of 24 younger children aged 7-8 years (mean age $7.58 \pm 0.50$ years; 14 girls) and 24 older children aged $11-12$ years (mean age $11.50 \pm 0.51$ years; 14 girls). Handedness was assessed with the Edinburgh Handedness Inventory (Oldfield, 1971). All participants were screened for pure-tone hearing thresholds $(\leq 35 \mathrm{~dB}$ HL; see Table 1$)$ and interaural threshold differences $(\leq 10 \mathrm{~dB})$ for the frequencies of 250, 500, 1000, 2000, and $3000 \mathrm{~Hz}$, using a pure-tone audiometer (MAICO Diagnostics MA 51, Berlin, Germany). None of the participants had to be excluded on the basis of hearing thresholds. Prior to the dichotic listening experiment, cognitive covariates including a marker of auditory working memory (auditory verbal $N$-back task; adapted from Lövdén, Schäfer, Pohlmeyer, \& Lindenberger, 2008) and a marker of perceptual speed (Digit Symbol Substitution test; Wechsler, 1981) were assessed. In line with previous developmental studies (e.g., Hämmerer, Li, Müller, \& Lindenberger, 2010; Vuontela et al., 2003), our results showed significant age-related improvements in auditory working memory performance, younger children, $11.9 \pm .9$, older children: $13.4 \pm$ $.6, t(46)=6.73, p<.05, d=1.94$; and perceptual speed, younger children, $26.9 \pm 5.6$, older children, $47.1 \pm 10.8, t(46)=6.73, p<$ $.05, d=2.34$, confirming the age typicality of our samples.

The adult development sample of the previously published study consisted of 24 right-handed younger adults aged 23-35 years (25.96 \pm 2.7 years; 12 women) and 25 right-handed older adults aged $65-76$ years (mean age $70.68 \pm 3.5$ years; 11 women). All participants had pure-tone hearing thresholds $\leq 35 \mathrm{~dB}$ HL (see Table 1) and interaural threshold differences $\leq 10 \mathrm{~dB}$ for the frequencies of 250, 500, 1000, 2000, and $3000 \mathrm{~Hz}$. More details about the adult development sample can be found in Passow et al. (2012).

The participants of the two studies were all native German speakers and were paid for participation. Before testing, informed consent was obtained from each participant and the parents of the participants, respectively. The Ethics Committee of the Max Planck Institute for Human Development, Berlin, Germany, approved both studies.

\section{Stimuli and Procedure}

The stimuli were paired presentations of CV syllables, which were formed combining the six stop-consonants $/ \mathrm{b}, \mathrm{d}, \mathrm{g}, \mathrm{p}, \mathrm{t}, \mathrm{k} /$ with the vowel /a/. The syllables were either voiced $(/ \mathrm{ba} /, / \mathrm{da} /$, $/ \mathrm{ga} /$ ) or unvoiced $(/ \mathrm{pa} /, / \mathrm{ta} /, / \mathrm{ka} /)$. All syllables were spoken by a young adult male speaker and with a mean duration of $400 \mathrm{~ms}$ and presented at individually adjusted intensities. Individual adjustment of intensities was done by adding a constant of $65 \mathrm{~dB}$ to each

Table 1

Pure-Tone Hearing Thresholds (db HL) for Younger Children, Older Children, Younger Adults, and Older Adults

\begin{tabular}{|c|c|c|c|c|c|c|c|c|}
\hline \multirow{2}{*}{$\begin{array}{l}\text { Frequency } \\
\text { in } \mathrm{Hz}\end{array}$} & \multicolumn{2}{|c|}{$\begin{array}{l}\text { Younger } \\
\text { children }\end{array}$} & \multicolumn{2}{|c|}{$\begin{array}{l}\text { Older } \\
\text { children }\end{array}$} & \multicolumn{2}{|c|}{$\begin{array}{l}\text { Younger } \\
\text { adults }\end{array}$} & \multicolumn{2}{|c|}{ Older adults } \\
\hline & $M$ & $S D$ & $M$ & $S D$ & $M$ & $S D$ & $M$ & $S D$ \\
\hline 250 & 4.79 & 5.31 & 5.21 & 4.94 & 6.25 & 4.10 & 10.70 & 6.48 \\
\hline 500 & 6.04 & 4.54 & 4.27 & 4.14 & 5.73 & 3.86 & 9.80 & 5.35 \\
\hline 1000 & 5.10 & 4.20 & 2.60 & 5.03 & 3.85 & 3.90 & 12.70 & 6.65 \\
\hline 2000 & 5.83 & 4.40 & 3.65 & 5.00 & 5.73 & 5.44 & 20.30 & 8.21 \\
\hline 3000 & 3.96 & 4.94 & 1.88 & 5.17 & 4.06 & 4.47 & 22.20 & 8.79 \\
\hline
\end{tabular}


participant's personal hearing threshold at $500 \mathrm{~Hz}$ (mean of $0-1000 \mathrm{~Hz}$ ), because the highest amplitude in all of the six CV syllables was present in the frequency range below $1000 \mathrm{~Hz}$. Prior to the experiment, a syllable discrimination task was conducted to ensure that the children in both age groups were able to discriminate between the six different syllables. The syllables were presented diotically, and the participants had to choose one out of six corresponding buttons (chance level 16.7\%). All children performed well above chance, and mean accuracy across both groups was $94.9 \pm 6.3 \%$.

Perceptual saliency of the syllables was manipulated by decreasing the intensity of either the right or the left ear in $5 \mathrm{~dB}$ steps until a maximum difference of $20 \mathrm{~dB}$ between ears was reached. Overall, there were nine different interaural intensity conditions: four conditions favoring the left ear, $\mathrm{L}>\mathrm{R}(1=[-20 \mathrm{~dB}], 2=[-15$ $\mathrm{dB}], 3=[-10 \mathrm{~dB}], 4=[-5 \mathrm{~dB}])$, four conditions favoring the right ear, $\mathrm{R}>\mathrm{L}(6=[5 \mathrm{~dB}], 7=[10 \mathrm{~dB}], 8=[15 \mathrm{~dB}], 9=[20$ $\mathrm{dB}]$ ), and one condition was neutral (same input intensity to both ears, $L=R(5=[0 \mathrm{~dB}])$. The neutral condition served as baseline intensity and was adapted to each participant's individual hearing threshold at $500 \mathrm{~Hz}$ (see above). Each of the 12 dichotic syllable pairs was presented twice for each of the nine perceptual saliency conditions, resulting in a total of 216 intensity-stimulus pairs for each attentional condition, which were then split into four testing blocks of 54 trials.

Attentional focus was manipulated by instructing the participants to attend to both ears (neutral-focus; NF) or to focus either on the right (focused-right; FR) or the left ear (focused-left; FL). In the NF condition, participants were asked to report the syllables they heard most clearly, whereas in the focused-attention conditions, they were asked to only report the syllable presented to the requested ear. The attentional instructions were varied across blocks. The NF condition was always completed first to avoid carryover effects from the FR or FL condition to the NF condition (Hiscock \& Stewart, 1984). FR and FL blocks were intermixed and individually counterbalanced in two presentation orders (i.e., $\mathrm{ABBABAAB}$ or BAABABBA). All testing was performed in a sound-attenuated booth. Presentation of the stimuli and response collection were controlled via E-prime v1.2 software (Psychology Software Tools) run on a PC. All stimuli were presented using insert earphones (ER 3A Insert Earphone, Etymotic Research, Inc., Elk Grove Village, IL).

\section{Statistical Analysis}

Extending previous studies focusing on the development of attentional control of dichotic listening (Hugdahl et al., 2001; Takio et al., 2009), we examined how the development of attentional control may interact with perceptual saliency to affect the laterality indices in an intensity- and attention-modulated dichotic listening task. In addition, we conducted secondary analyses comparing variables of interest of the present study with corresponding data from an analog study testing an adult development sample (Passow et al., 2012) to investigate potential similarities and differences between child development and aging.

Child developmental effects in the interaction between perceptual saliency and attentional focus were analyzed with respect to the auditory laterality index, a summary measure of ear advantage. The laterality index represents the number of correct right-ear (RE) reports in relation to the correct left-ear (LE) reports (i.e., ((RE $\mathrm{LE}) /(\mathrm{RE}+\mathrm{LE}) \times 100)$. The laterality indices for younger and older children were analyzed in a repeated measures analysis of variance (ANOVA), with attentional focus (NF, FR, FL) and perceptual saliency (nine interaural intensity difference conditions) as within-subject factors and age group and sex as between-subject factors.

For the secondary data analyses of life span comparisons, first the presence of a reliable REA in younger and older children of the present study and younger and older adults from an earlier study (Passow et al., 2012) was analyzed with respect to the percentage of reported RE and LE syllables in the neutral-focus condition when inputs to both ears were of the same intensity. Correct reports were analyzed in a repeated measures ANOVA, with ear (RE, LE) as the within-subject factor and age group and sex as between-subject factors. Second, to explore child development and aging-related influences on the interaction between perceptual saliency and attentional control during auditory processing, we computed a Selective Attention Index (ATTIndex) and a Perceptual Dependency Index (PERIndex) for each participant of the four age groups. The ATTIndex reflects the extent to which participants were able to deploy their attention flexibly to focus either on the $\mathrm{RE}$ or LE inputs. The ATTIndex was based on the increase of RE reports in the FR condition and the increase in LE reports in the FL condition relative to the NF condition. For each of the nine levels of perceptual saliency, the ATTIndex was computed by first calculating the differences between (a) the laterality indices in FR and $\mathrm{NF}$ conditions (for an illustration, see differences between the dotted and solid lines in Figure 1B) and (b) the laterality indices in $\mathrm{NF}$ and FL conditions (for an illustration, see differences between the solid and dashed lines in Figure 1B). The resulting differences were then summed across the nine levels of perceptual saliency for the FR and FL conditions. In order to get an overall index of attentional flexibility, the mean of the summed differences for FR and FL was taken as the ATTIndex (for equation, see the Appendix). High values of the ATTIndex reflect great increases in reporting stimuli from the attended ear in the FR and FL relative to the NF condition and, thus, high attentional flexibility. The PERIndex reflects the extent to which the performance was driven by perceptual saliency of the syllables. At the first step, a PERIndex was computed separately for each of the three attention conditions (FR, FL, and NF). This was done by calculating the mean of the absolute differences between the neutral condition (the condition with $0 \mathrm{~dB}$ difference in Figure 1B; i.e., when both ears get the same input intensity), and each of the four perceptual saliency conditions favoring either the right or the left ear. At the second step, an overall perceptual dependency index was defined as the mean of the three perceptual dependency indices (for equations, see the Appendix). High values of the PERIndex indicate increased reports of the louder relative to the softer syllable and, thus, high dependency on bottom-up stimulus salience.

Life span age differences in both indices were analyzed in two separate univariate ANOVAs, with age group and sex as betweensubject factors. Given the improvements in auditory attentional control during child development and the decline in auditory attentional control during aging (Hugdahl et al., 2001; Takio et al., 2009), significant main effects of age group in the ATTIndex were followed up by a planned contrast testing for a curvilinear pattern. In contrast, as auditory perceptual processing progressively declines 
throughout the life span (see Gordon-Salant, Frisina, Popper, \& Fay, 2010, for a review), significant main effects in PERIndex were followed up by planned contrasts testing for a linear pattern. GamesHowell pairwise comparisons, which correct for Type I error accumulation and are suitable for samples with unequal variances, were calculated to further characterize the age group differences in both indices. Furthermore, Pearson product-moment correlations were used to analyze whether the magnitude of the REA, expressed in the laterality index, is associated with the ATTIndex or PERIndex.

Given that sex as a between-subject factor did not reveal any significant main or interaction effects, this factor was collapsed in all subsequent ANOVAs. When the sphericity assumption was violated $(p<.05)$, the Greenhouse-Geisser correction was applied. Adjusted degrees of freedom and $p$ values of the analyses are reported. Effect sizes of main or interaction effects are given as $\eta^{2}$, representing the proportion of variance of the dependent factor explained by the independent variable. Effect sizes of follow-up planned contrasts were given as Cohen's $d$. The alpha level was set to $p=.05$.

\section{Results}

\section{Child Developmental Differences in the Interplay Between Perceptual Saliency and Attentional Focus}

The results of the three-way repeated measures ANOVA analyzing the laterality indices revealed significant main two- and three-way interaction effects (see Table 2). Most importantly, the three-way interaction of attentional focus, perceptual saliency, and age group reached significance, $F(8.13,373.98)=3.44, p<.05$, $\eta^{2}=0.01$, indicating age differences in the interaction between perceptual saliency and attentional focus during auditory processing (see Figure 2). Results of the post hoc attentional Focus $\times$ Perceptual Saliency ANOVAs conducted separately for each age group demonstrated a significant main effect of perceptual saliency in both age groups, younger children, $F(1.76,40.56)=73.95, p<.05$, $\eta^{2}=0.86$, and older children, $F(1.82,41.90)=82.75, p<.05, \eta^{2}=$ 0.68 . In contrast, the main effect of attentional focus was observed in older children, $F(1.12,25.65)=14.49, p<.05, \eta^{2}=0.23$, but not in younger children $(p>.05)$. In older children, the laterality index in the FR condition was significantly larger than the laterality index in the NF condition when perceptual saliency favored the left ear (i.e., 20 $\mathrm{dB}, 10 \mathrm{~dB}$, and $5 \mathrm{~dB}$ in favor of the left ear) and in one condition in which perceptual saliency and attentional focus favored the same ear (i.e., $5 \mathrm{~dB}$ in favor of the right ear). In younger children, none of the

Table 2

Three-Way Repeated Measures ANOVA for Laterality Indices

\begin{tabular}{lrcrc}
\hline \multicolumn{1}{c}{ Effect } & \multicolumn{1}{c}{$F$} & $d f$ effect & $d f$ error & $\eta^{2}$ \\
\hline ATT & 15.29 & 1.24 & 57.22 & 0.08 \\
ATT $\times$ Age Group & 9.82 & 1.24 & 57.22 & 0.05 \\
PER & 155.33 & 1.88 & 86.86 & 0.73 \\
ATT $\times$ PER $\times$ Age Group & 3.44 & 8.13 & 373.98 & 0.01 \\
\hline
\end{tabular}

Note. Attentional focus (ATT) and perceptual saliency (PER) are withinsubject factors, and age group is a between-subject factor. ANOVA = analysis of variance. All effects were statistically significant at $p<.05$. comparisons reached significance. Regarding FL conditions, older children showed a significantly lower laterality index relative to the NF condition in three conditions in which perceptual saliency and attentional focus were in opposition (i.e., $20 \mathrm{~dB}, 15 \mathrm{~dB}$, and $5 \mathrm{~dB}$ in favor of the right ear) and in one condition when both favored the same ear (i.e., $15 \mathrm{~dB}$ in favor of the left ear). Again, no such differences were observed in the younger children.

These results indicate that the behavior of both younger and older children was influenced by the difference in perceptual saliency between left and right auditory inputs. However, older children's behavior was also significantly influenced by attentional control, whereas younger children's behavior was not. In older children, the effect of attentional focus on the REA increased when perceptual saliency and attentional focus favored opposing ears relative to conditions in which both favored the same ear.

\section{Life Span Differences in Attentional Control and Dependency on Perceptual Saliency}

The results of the two-way repeated measures ANOVA analyzing the percentages of correct RE and LE reports in the NF condition when both ears get the same input intensity revealed a main effect of ear, $F(1,89)=30.83, p<.05, \eta^{2}=0.65$, and age group, $F(1,89)=8.62, p<.05, \eta^{2}=0.05$. The interaction between ear and age group did not reach significance $(p>.05)$. Follow-up paired samples $t$ tests within each age group revealed a pronounced REA in younger, $t(23)=3.93, p<.05, d=.74$, and older children, $t(23)=3.34, p<.05, d=1.14$, as well as in younger adults, $t(23)=4.67, p<.05, d=1.34$. In contrast, older adults did not report more syllables from the right compared with the left ear $(p>.05)$. Across all four age groups, the strength of the REA was associated neither with the ability to exert attention flexibly (i.e., the ATTIndex, $r=-.002, p>.05$ ) nor with the dependency on stimulus saliency (i.e., the PERIndex, $r=-.118$, $p>.05$ ), and also not when controlled for age (ATTIndex: $r=$ $.001, p>.05$; PERIndex: $r=-.112, p>.05)$.

The life span comparison of the ATTIndex and the PERIndex revealed a significant main effect of age group in both indices, ATTIndex, $F(3,93)=7.99, p<.05, \eta^{2}=0.20$; PERIndex, $F(3$, $93)=8.62, p<.05, \eta^{2}=0.22$ (see Figure $3 \mathrm{~B}$ ). Regarding the ATTIndex, planned contrasts confirmed a curvilinear pattern across age groups, with younger children and older adults having the lowest ATTIndex, $t(56.47)=-4.75, p<.05, d=1.26$. Games-Howel pairwise comparisons revealed that younger children showed a significantly lower ATTIndex than older children (mean difference: $-17.45 \%, p<.05, d=0.96$ ) and younger adults (mean difference: $-22.44 \%, p<.05, d=1.17$ ). Older children and younger adults did not differ with respect to their ATTIndex (all $p s>$.05). In contrast, the ATTIndex of older adults was significantly lower than that of older children (mean difference: $-14.23 \%, p<.05, d=0.76$ ) and younger adults (mean difference: $-19.22 \%, p<.05, d=0.98$ ), but did not differ from that of younger children.

With respect to the dependency on perceptual saliency of the syllables captured by the PERIndex, the planned contrasts confirmed a linear pattern, with older adults showing the highest value, $t(93)=4.69, p<.05, d=0.97$. Pairwise comparisons showed that younger children did not significantly differ from older children and younger adults (all $p \mathrm{~s}>.05$ ). In contrast, the 
A

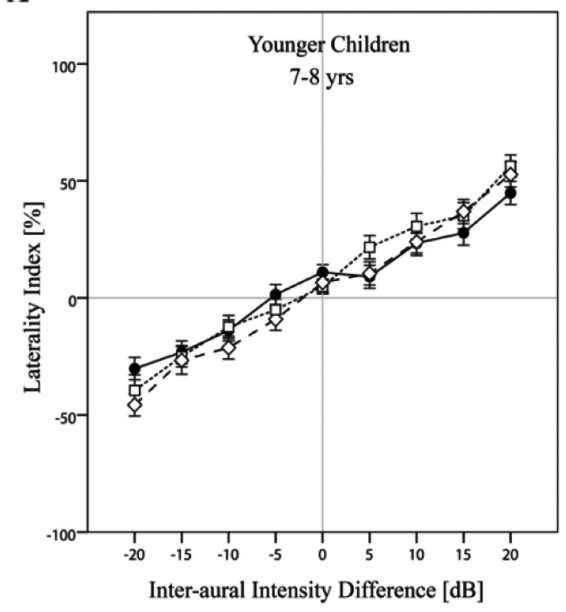

B

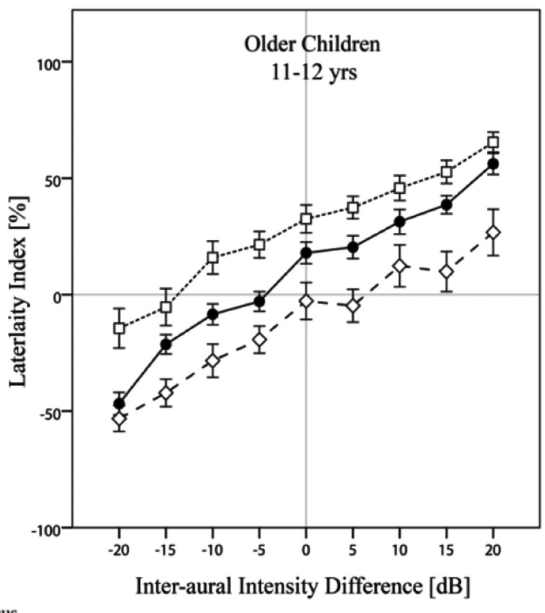

Figure 2. Mean laterality indices across all interaural intensity difference conditions and for each attentional focus condition for (A) younger children and (B) older children. Error bars indicate one standard error of the mean.

PERIndex of older adults was significantly higher than the PERIndex of younger children (mean difference: $10.44 \%, p<.05$, $d=1.04$ ), older children (mean difference: $11.35 \%, p<.05, d=$ 1.29), and younger adults (mean difference: 6.68\%, $p<.05, d=$ $0.75)$. In sum, younger children's performance disadvantage relative to older children and younger adults was primarily attributable to immature attentional control, whereas older adults' performance in dichotic listening was, in addition, strongly driven by the perceptual saliency of the syllables.

\section{Discussion}

The age group differences observed in the present study are consistent with the hypothesis that the interaction between perceptual saliency and attentional control during auditory processing changes from middle to late childhood. Extending the existing dichotic listening literature on child development of auditory attentional control, the present study showed that older children aged 11-12 years were able to focus their attention flexibly on either ear, even when they were confronted with perceptually more salient irrelevant information from the nonattended ear. In contrast, younger children aged 7-8 years were unable to do so. Moreover, we also compared the childhood findings with data from a sample of healthy younger and older adults (Passow et al., 2012) on indices of selective attention and perceptual dependency and found that the interaction between perceptual saliency and attentional control differed across the life span. In the following, we further discuss the similarities and differences between child development and healthy aging.

\section{Attentional Control of Auditory Processing Increased From Middle to Late Childhood}

On the basis of the present findings, it appears that perceptual saliency and attentional focus interact in both younger and older children. However, the pattern of this interaction evolves with age. Whereas older children were able to focus their attention flexibly on the right or left ear even when they were confronted with perceptually more salient stimuli from the nonattended ear, younger children apparently had difficulties in doing so. Specifically, across all perceptual saliency conditions, in older children the laterality indices were increased relative to the NF condition when attending to the right ear; conversely, attending to the left ear reduced the laterality indices. Furthermore, these effects were enhanced in conditions in which attentional focus and perceptual saliency favored opposing ears, documenting the influence of top-down control on auditory perception. In contrast, the laterality indices in younger children differ much less between the three attentional focus conditions. Taken together, these results indicate that, compared with younger children, older children deployed attentional control more effectively and more flexibly in modulating the laterality indices.

In light of the three-network view of attention (Posner \& Boies, 1971) that differentiates between an alerting, orienting, and executive attention network, our results are in line with the development of the executive attention network. This network is commonly regarded as a supervisory system that resolves conflicts among different competing stimuli and is involved in planning and decision-making processes (e.g., Fan, McCandliss, Sommer, Raz, \& Posner, 2002; Posner \& Petersen, 1990; Raz \& Buhle, 2006). In the present intensity- and attention-modulated dichotic listening paradigm, executive attention is needed to resolve conflicts between exogenous (bottom-up) orienting when attention is captured automatically by the perceptually more salient stimuli and endogenous (top-down) orienting when attention is focused voluntarily on either ear. Developmental studies have shown a lack of executive attention in early childhood and progressive improvements during child development in visual (e.g., Ridderinkhof et al., 1997; Rueda et al., 2004; Waszak et al., 2010) and auditory attention 
A

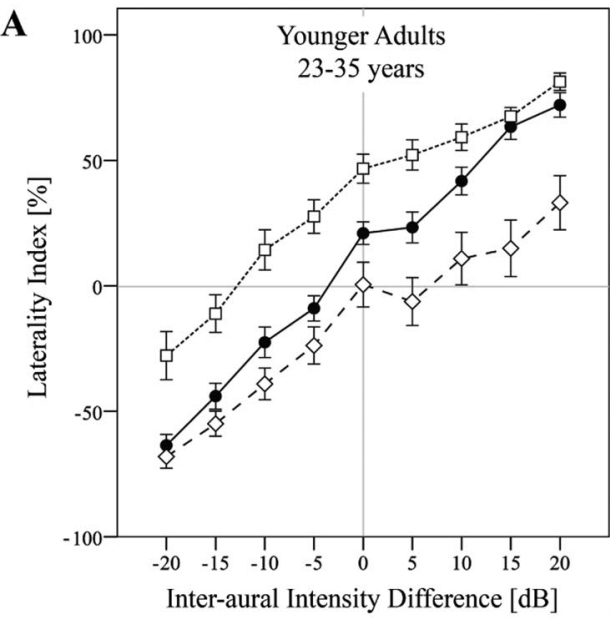

B

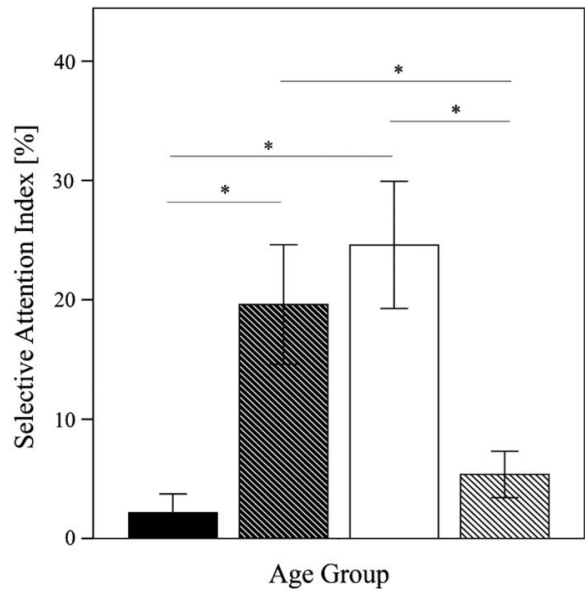

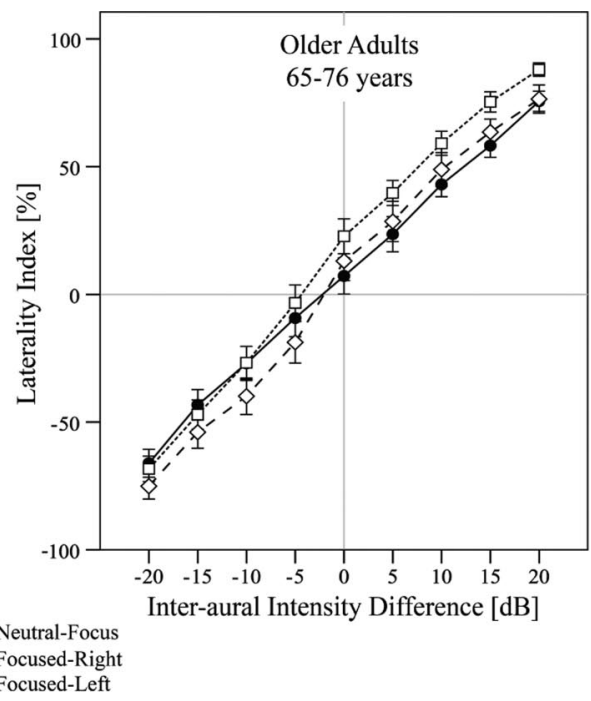

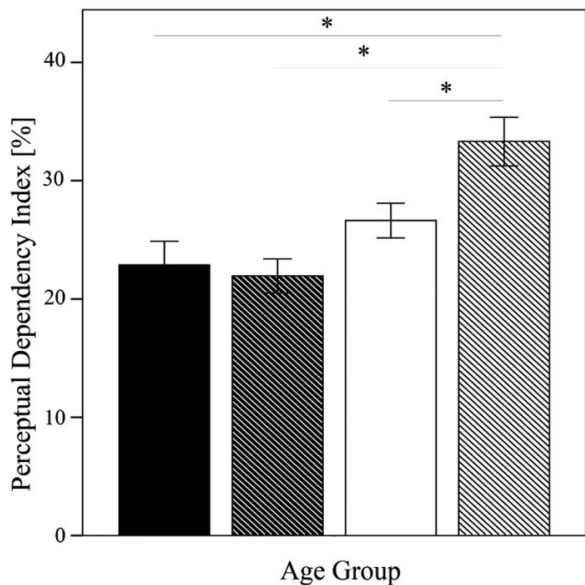

Age Group

Younger Children

N Older Children

$\square$ Younger Adults

Older Adults

Figure 3. A: Mean laterality indices across all interaural intensity difference conditions and for each attentional focus condition for younger adults (left panel) and older adults (right panel). B: Mean Selective Attention Index (left panel) and mean Perceptual Dependency Index (right panel) across the four age groups. Error bars indicate one standard error of the mean. ${ }^{*} p<.05$. Error bars indicate $1 S E$ of the mean. A is adapted from "Human Aging Compromises Attentional Control of Auditory Perception," by S. Passow, R. Westerhausen, I. Wartenburger, K. Hugdahl, H. R. Heekeren, U. Lindenberger U, and S.-C. Li, 2012, Psychology and Aging, 27 p. 102. Copyright 2012 by the American Psychological Association.

tasks (e.g., Gomes et al., 2000; Hugdahl et al., 2001; Takio et al., 2009). For instance, interference effects in the Eriksen Flanker task, as reflected in decrements in response speed and accuracy when incongruent response options have to be suppressed, decrease with increasing age in childhood (Li et al., 2009; Ridderinkhof et al., 1997; Rueda et al., 2004). Furthermore, our results confirm previous findings obtained with forced-attention dichotic listening paradigms showing that the ability to focus attention on either ear and suppress the input of the nonattended ear improves during child development (Hugdahl et al., 2001; Takio et al., 2009).
Previous functional magnetic resonance imaging (Falkenberg, Specht, \& Westerhausen, 2011; Westerhausen et al., 2010) and electroencephalography (Passow et al., 2012) studies suggest that a network of frontal and parietal regions is involved in the intensity- and attention-modulated dichotic listening task. The maturation-driven synaptogenesis of parietal structures is likely to asymptote at about 10 years of age (Casey, Tottenham, Liston, \& Durston, 2005), whereas synaptogenesis and other maturational changes in the prefrontal cortex continue into and beyond adolescence (Sowell et al., 2003). Relating these findings with our data, one viable interpretation is that immature parietal and prefrontal 
networks in younger children may contribute to their difficulties in efficiently and flexibly exerting attention in the context of the present task.

According to Posner and Rothbart (1998), the development of executive attention is closely intertwined with the development from rudimentary, rigid to conscious, intentional controls of ones' own thoughts, emotions, and actions (i.e., self-regulation). For instance, measures of conflict resolution significantly correlate with children's self-regulation in daily life measured by parental reports (González, Fuentes, Carranza, \& Estévez, 2001; Posner \& Rothbart, 2009). Thus, the observed performance increase in attentional control of auditory perception between younger and older children may also be seen in light of the development from reactive to more self-regulative behavior. Furthermore, individual differences in the ability to focus on relevant information and to suppress irrelevant, perceptually more salient information may be closely related to individual differences in learning at schools. Thus, future studies should investigate whether students with attentional deficits can be trained on their performance in the intensity- and attention-modulated dichotic listening paradigm and whether the training effect would influence their school achievements.

\section{Similarities and Differences Between Middle Childhood and Old Age}

In line with the literature, a reliable RE advantage could be observed in younger and older children (Berlin et al., 1973; Harper \& Kraft, 1994; Westerhausen et al., 2010), as well as in younger adults (for a review, see Hugdahl, 2003; Kimura, 1967). The absence of an RE advantage in older adults can be seen in light of a more bilateralized functional language network (Tyler et al., 2010) and might reflect age-related differences in distinctiveness of brain activations (Li \& Sikstöm, 2002). The strength of the RE advantage was not associated with attentional flexibility or dependency on stimulus saliency. This indicates that attentional flexibility and perceptual dependency observed in this study are not specifically linked to mechanisms that underlie the REA.

Comparing indices of selective attention and perceptual dependency obtained from the current child development sample with data from a previously published adult sample revealed informative similarities and differences between age-graded changes in childhood and adulthood. At first glance, age differences in the effects of attentional focus on laterality appear in children and adults (i.e., comparing the younger vs. older children data in Figure 2 and the older vs. younger adult data in Figure 3A). Both, in younger children and older adults, the laterality indices were not modulated by attentional focus across all levels of perceptual saliency. In contrast, older children's and younger adults' laterality indices in the FR or FL conditions differed substantially from those in the NF conditions, reflecting the flexible and efficient deployment of attentional control. The same pattern of findings holds for the selective attention indices, which are reliably lower in younger children and older adults than in older children and younger adults, respectively (see Figure 3B). On the basis of these findings, we conclude that auditory attentional control follows an inverted U-shaped function, in line with earlier evidence on the life span development of visual attention (Hommel, Li, \& Li, 2004; Waszak et al., 2010), inhibition (Dempster, 1992), working mem- ory (Sander, Werkle-Bergner, \& Lindenberger, 2011), and episodic memory (Shing \& Lindenberger, 2011).

At the same time, we observed important differences in perceptual dependency between children and older adults. Among the four age groups, the group of older adults showed the highest perceptual dependency, whereas among younger adults, older and younger children did not differ significantly from each other (see Figure 3B). Available evidence points toward a stability in hearing sensitivity from childhood to young adulthood and a progressive decline thereafter (e.g., Cruickshanks et al., 1998; Trehub et al., 1988). As hearing sensitivity and attentional control both decline during aging, the processing of competing simultaneous auditory inputs may increasingly depend on the relative saliency of auditory inputs. On top of their attentional deficit, older adults' dichotic listening performance was more dependent on perceptual saliency than the listening performance in younger adults and children.

\section{Conclusion}

The present study provides novel evidence for child developmental differences in the interaction between perceptual saliency and attentional control during auditory processing. Our results showed that younger and older children's behavior reflects, to some extent, perceptual saliency differences of the auditory inputs between the ears. Older children are able to flexibly exert their attention to overcome irrelevant auditory information of different perceptual saliency, whereas younger children are not yet able to do so in the context of the present paradigm. Critically, direct comparisons of these results with previous findings obtained from an adult development sample (Passow et al., 2012) revealed a clear life span dissociation: Younger children's performance restrictions reflect difficulties in attentional control only, whereas older adults' performance deficits reflect both difficulties in attentional control and an exaggerated reliance on perceptual saliency. In line with previous studies (e.g., Hämmerer et al., 2011; Li et al., 2009; Sander et al., 2011; Shing \& Lindenberger, 2011; Waszak et al., 2009), our results further reveal that child development and aging are not simple mirror reflections of each other and underscore the importance of life span comparisons. The generality of our findings, however, needs to be verified in future studies with larger samples as well as in studies using longitudinal designs.

\section{References}

Baltes, P. B., Lindenberger, U., \& Staudinger, U. M. (2006). Life-span theory in developmental psychology. In W. Damon \& R. W. Lerner (Eds.), Handbook of child psychology: Theoretical models of human development (6th ed., Vol. 1, pp. 569-664). New York, NY: Wiley.

Berlin, C. I., Lowe-Bell, S., Hughes, L. F., \& Berlin, H. L. (1973). Dichotic right ear advantage in males and females-ages 5 to 13. Journal of the Acoustical Society of America, 53, 368. doi:10.1121/1.1982639

Casey, B. J., Tottenham, N., Liston, C., \& Durston, S. (2005). Imaging the developing brain: What have we learned about cognitive development? Trends in Cognitive Sciences, 9, 104-110. doi:10.1016/j.tics.2005.01 .011

Craik, F. I. M., \& Bialystok, E. (2006). Cognition through the lifespan: Mechanisms of change. Trends in Cognitive Sciences, 10, 131-138. doi:10.1016/j.tics.2006.01.007

Cruickshanks, K. J., Wiley, T. L., Tweed, T. S., Klein, B. E., Klein, R., Mares-Perlman, J. A., \& Nondahl, D. (1998). Prevalence of hearing loss in older adults in Beaver Dam, Wisconsin: The Epidemiology of Hearing 
Loss Study. American Journal of Epidemiology, 148, 879-886. doi: 10.1093/oxfordjournals.aje.a009713

Dempster, F. N. (1992). The rise and fall of the inhibitory mechanism: Toward a unified theory of cognitive development and aging. Developmental Review, 12, 45-75. doi:10.1016/0273-2297(92)90003-K

Desimone, R., \& Duncan, J. (1995). Neural mechanisms of selective visual-attention. Annual Review of Neuroscience, 18, 193-222. doi: 10.1146/annurev.ne.18.030195.001205

Eriksen, B. A., \& Eriksen, C. W. (1974). Effects of noise letters upon identification of a target letter in a nonsearch task. Perception \& Psychophysics, 16, 143-149. doi:10.3758/BF03203267

Falkenberg, L. E., Specht, K., \& Westerhausen, R. (2011). Attention and cognitive control networks assessed in a dichotic listening fMRI study. Brain and Cognition, 76, 276-285. doi:10.1016/j.bandc.2011.02.006

Fan, J., McCandliss, B. D., Sommer, T., Raz, A., \& Posner, M. I. (2002). Testing the efficiency and independence of attentional networks. Journal of Cognitive Neuroscience, 14, 340-347. doi:10.1162/ 089892902317361886

Gomes, H., Molholm, S., Christodoulou, C., Ritter, W., \& Cowan, N. (2000). The development of auditory attention in children. Frontiers in Bioscience, 5, D108-D120. doi:10.2741/Gomes

González, C., Fuentes, L. J., Carranza, J. A., \& Estévez, A. F. (2001). Temperament and attention in the self-regulation of 7-year-old children. Personality and Individual Differences, 30, 931-946. doi:10.1016/ S0191-8869(00)00084-2

Gordon-Salant, S., Frisina, D. R., Popper, A. N., \& Fay, R. R. (2010). The aging auditory system. New York, NY: Springer. doi:10.1007/978-14419-0993-0

Hämmerer, D., Li, S. C., Müller, V., \& Lindenberger, U. (2010). An electrophysiological study of response conflict processing across the lifespan: Assessing the roles of conflict monitoring, cue utilization, response anticipation, and response suppression. Neuropsychologia, 48, 3305-3316. doi:10.1016/j.neuropsychologia.2010.07.014

Hämmerer, D., Li, S. C., Müller, V., \& Lindenberger, U. (2011). Life span differences in electrophysiological correlates of monitoring gains and losses during probabilistic reinforcement learning. Journal of Cognitive Neuroscience, 23, 579-592. doi:10.1162/jocn.2010.21475

Harper, L. V., \& Kraft, R. H. (1994). Longitudinal change of ear advantage for receptive language function in 21/2 to 9-year-old children. Perceptual and Motor Skills, 79, 1091-1102. doi:10.2466/pms.1994.79.3.1091

Helfer, K. S., \& Freyman, R. L. (2008). Aging and speech-on-speech masking. Ear and Hearing, 29, 87-98.

Hiscock, M., \& Stewart, C. (1984). The effect of asymmetrically focused attention upon subsequent ear differences in dichotic listening. Neuropsychologia, 22, 337-351. doi:10.1016/0028-3932(84)90080-0

Hommel, B., Li, K. Z. H., \& Li, S. C. (2004). Visual search across the life span. Developmental Psychology, 40, 545-558. doi:10.1037/0012-1649 .40 .4 .545

Hugdahl, K. (2003). Dichotic listening in the study of auditory laterality. In K. Hugdahl \& R. J. Davidson (Eds.), The asymmetrical brain (pp. 441-476). Cambridge, MA: MIT Press. doi:10.1016/j.bandc.2008.02 .106

Hugdahl, K., Carlsson, G., \& Eichele, T. (2001). Age effects in dichotic listening to consonant-vowel syllables: Interactions with attention. Developmental Neuropsychology, 20, 445-457. doi:10.1207/ S15326942DN2001_8

Kail, R., \& Salthouse, T. A. (1994). Processing speed as a mental-capacity. Acta Psychologica, 86, 199-225. doi:10.1016/0001-6918(94)90003-5

Kimura, D. (1967). Functional asymmetry of the brain in dichotic listening. Cortex, 3, 163-178.

Li, S-C., Hämmerer, D., Müller, V., Hommel, B., \& Lindenberger, U. (2009). Lifespan development of stimulus-response conflict cost: Similarities and differences between maturation and senescence. Psychological Research, 73, 777-785. doi:10.1007/s00426-008-0190-2
Li, S-C., Lindenberger, U., Hommel, B., Aschersleben, G., Prinz, W., \& Baltes, P. B. (2004). Transformations in the couplings among intellectual abilities and constituent cognitive processes across the life span Psychological Science, 15, 155-163. doi:10.1111/j.0956-7976.2004 .01503003.x

Li, S-C., \& Sikström, S. (2002). Integrative neurocomputational perspectives on cognitive aging, neuromodulation and representation. Neuroscience and Biobehavioral Reviews, 26, 795-808. doi:10.1016/S01497634(02)00066-0

Lövdén, M., Schäfer, S., Pohlmeyer, A. E., \& Lindenberger, U. (2008). Walking variability and working-memory load in aging: A dual-process account relating cognitive control to motor control performance. Journals of Gerontology Series B: Psychological Sciences and Social Sciences, 63, P121-P128. doi:10.1093/geronb/63.3.P121

Marshall, J. C., Caplan, D., \& Holmes, J. M. (1975). Measure of laterality. Neuropsychologia, 13, 315-321. doi:10.1016/0028-3932(75)90008-1

Miller, E. K., \& Cohen, J. D. (2001). An integrative theory of prefrontal cortex function. Annual Review of Neuroscience, 24, 167-202. doi: 10.1146/annurev.neuro.24.1.167

Oldfield, R. C. (1971). Assessment and analysis of handedness: The Edinburgh Inventory. Neuropsychologia, 9, 97-113. doi:10.1016/00283932(71)90067-4

Passow, S., Westerhausen, R., Hugdahl, K., Wartenburger, I., Heekeren, H. R., Lindenberger, U., \& Li, S.-C. (2012). Electrophysiological correlates of adult age differences in attentional control of auditory processing. Cerebral Cortex. doi:10.1093/cercor/bhs306

Passow, S., Westerhausen, R., Wartenburger, I., Hugdahl, K., Heekeren, H. R., Lindenberger, U., \& Li, S.-C. (2012). Human aging compromises attentional control of auditory perception. Psychology and Aging, 27, 99-105. doi:10.1037/a0025667

Posner, M. I., \& Boies, S. J. (1971). Components of attention. Psychological Review, 78, 391-408. doi:10.1037/h0031333

Posner, M. I., \& Petersen, S. E. (1990). The attention system of the human brain. Annual Review of Neuroscience, 13, 25-42. doi:10.1146/annurev .ne.13.030190.000325

Posner, M. I., \& Rothbart, M. K. (1998). Attention, self-regulation and consciousness. Philosophical Transactions of the Royal Society B: Biological Sciences, 353, 1915-1927. doi:10.1098/rstb.1998.0344

Posner, M. I., \& Rothbart, M. K. (2009). Toward a physical basis of attention and self-regulation. Physics of Life Reviews, 6, 103-120. doi: 10.1016/j.plrev.2009.02.001

Raz, A., \& Buhle, J. (2006). Typologies of attentional networks. Nature Reviews Neuroscience, 7, 367-379. doi:10.1038/nrn1903

Ridderinkhof, K. R., van der Molen, M. W., Band, G. P. H., \& Bashore, T. R. (1997). Sources of interference from irrelevant information: A developmental study. Journal of Experimental Child Psychology, 65, 315-341. doi:10.1006/jecp.1997.2367

Rueda, M. R., Fan, J., McCandliss, B. D., Halparin, J. D., Gruber, D. B., Lercari, L. P., \& Posner, M. I. (2004). Development of attentional networks in childhood. Neuropsychologia, 42, 1029-1040. doi:10.1016/ j.neuropsychologia.2003.12.012

Sander, M. C., Werkle-Bergner, M., \& Lindenberger, U. (2011). Binding and strategic selection in working memory: A lifespan dissociation. Psychology and Aging, 26, 612-624. doi:10.1037/a0023055

Shing, Y. L., \& Lindenberger, U. (2011). The development of episodic memory: lifespan lessons. Child Development Perspectives, 5, 148-155 doi:10.1111/j.1750-8606.2011.00170.x

Sowell, E. R., Peterson, B. S., Thompson, P. M., Welcome, S. E., Henkenius, A. L., \& Toga, A. W. (2003). Mapping cortical change across the human life span. Nature Neuroscience, 6, 309-315. doi:10.1038/nn1008

Stalinski, S. M., Schellenberg, E. G., \& Trehub, S. E. (2008). Developmental changes in the perception of pitch contour: Distinguishing up from down. Journal of the Acoustical Society of America, 124, 17591763. doi: $10.1121 / 1.2956470$ 
Sussman, E., Wong, R., Horvath, J., Winkler, I., \& Wang, W. (2007). The development of the perceptual organization of sound by frequency separation in 5-11-year-old children. Hearing Research, 225, 117-127. doi:10.1016/j.heares.2006.12.013

Takio, F., Koivisto, M., Jokiranta, L., Rashid, F., Kallio, J., Tuominen, T., . . Hämäläinen, H. (2009). The effect of age on attentional modulation in dichotic listening. Developmental Neuropsychology, 34, 225-239. doi: $10.1080 / 87565640902805669$

Tallus, J., Hugdahl, K., Alho, K., Medvedev, S., \& Hamalainen, H. (2007). Interaural intensity difference and ear advantage in listening to dichotic consonant-vowel syllable pairs. Brain Research, 1185, 195-200. doi: 10.1016/j.brainres.2007.09.012

Trehub, S. E., Bull, D., \& Thorpe, L. A. (1984). Infants' perception of melodies: The role of melodic contour. Child Development, 55, 821830. doi: $10.2307 / 1130133$

Trehub, S. E., Schneider, B. A., Morrongiello, B. A., \& Thorpe, L. A. (1988). Auditory-sensitivity in school-age children. Journal of Experimental Child Psychology, 46, 273-285. doi:10.1016/0022-0965(88)90060-4

Trehub, S. E., Thorpe, L. A., \& Morrongiello, B. A. (1987). Organizational processes in infants' perception of auditory patterns. Child Development, 58, 741-749. doi:10.2307/1130211

Tun, P. A., O'Kane, G., \& Wingfield, A. (2002). Distraction by competing speech in young and older adult listeners. Psychology and Aging, 17, 453-467. doi:10.1037/0882-7974.17.3.453

Tyler, L. K., Shafto, M. A., Randall, B., Wright, P., Marslen-Wilson, W. D., \& Stamatakis, E. A. (2010). Preserving syntactic processing across the adult life span: The modulation of the frontotemporal language system in the context of age-related atrophy. Cerebral Cortex, 20, 352-364. doi:10.1093/cercor/bhp105
Vuontela, V., Steenari, M-R., Carlson, S., Koivisto, J., Fjällberg, M., \& Aronen, E. T. (2003). Audiospatial and visuospatial working memory in 6-13 year old school children. Learning \& Memory, 10, 74-81. doi: $10.1101 / 1 \mathrm{~m} .53503$

Waszak, F., Li, S. C., \& Hommel, B. (2010). The development of attentional networks: Cross-sectional findings from a life span sample. Developmental Psychology, 46, 337-349. doi:10.1037/a0018541

Waszak, F., Schneider, W. X., Li, S. C., \& Hommel, B. (2009). Perceptual identification across the life span: A dissociation of early gains and late losses. Psychological Research, 73, 114-122. doi:10.1007/s00426-0080139-5

Wechsler, D. (1981). Wechsler Adult Intelligence Scale-revised. New York, NY: Psychological Corporation.

Werner, L. A. (1996). The development of auditory behavior (or what the anatomists and physiologists have to explain). Ear and Hearing, 17, 438-446. doi:10.1097/00003446-199610000-00010

Westerhausen, R., Helland, T., Ofte, S., \& Hugdahl, K. (2010). A longitudinal study of the effect of voicing on the dichotic listening ear advantage in boys and girls at age 5 to 8. Developmental Neuropsychology, 35, 752-761. doi:10.1080/87565641.2010.508551

Westerhausen, R., Moosmann, M., Alho, K., Belsby, S.-O., Hämäläinen, H., Medvedev, S., . . Hugdahl, K. (2010). Identification of attention and cognitive control networks in a parametric auditory fMRI study. Neuropsychologia, 48, 2075-2081. doi:10.1016/j.neuropsychologia.2010.03 .028

Westerhausen, R., Moosmann, M., Alho, K., Medvedev, S., Hamalainen, H., \& Hugdahl, K. (2009). Top-down and bottom-up interaction: Manipulating the dichotic listening ear advantage. Brain Research, 1250, 183-189. doi:10.1016/j.brainres.2008.10.070 


\section{Appendix}

\section{Equations of Selective Attention Index and Perceptual Dependency Index}

Selective Attention Index (ATTIndex) was defined as:

$$
\text { ATTIndex }=\frac{\sum_{I A I=1}^{I A I=9}\left(L I_{I A I} F R-L I_{I A I} N F\right)+\sum_{I A I=1}^{I A I=9}\left(L I_{I A I} N F-L I_{I A I} F L\right)}{9}
$$

Perceptual Dependency Index (PERIndex) was defined as:

$$
\begin{aligned}
\text { PERIndex } N F= & \frac{\sum_{I A I=1}^{I A I=4} A B S\left(A B S\left(L I_{I A I 5} N F\right)-A B S\left(L I_{I A I} N F\right)\right)}{4}+\frac{\sum_{I A I=6}^{I A I=9} A B S\left(A B S\left(L I_{I A I 5} N F\right)-A B S\left(L I_{I A I} N F\right)\right)}{4} \\
\text { PERIndexFR }= & \frac{\sum_{I A I=1}^{I A I=4} A B S\left(A B S\left(L I_{I A I 5} F R\right)-A B S\left(L I_{I A I} F R\right)\right)}{4}+\frac{\sum_{I A I=6}^{I A I=9} A B S\left(A B S\left(L I_{I A I 5} F R\right)-A B S\left(L I_{I A I} F R\right)\right)}{4} \\
\text { PERIndexFL }= & \frac{\sum_{I A I=1}^{I A I=4} A B S\left(A B S\left(L I_{I A I 5} F L\right)-A B S\left(L I_{I A I} F L\right)\right)}{4}+\frac{\sum_{I A I=6}^{I A I=9} A B S\left(A B S\left(L I_{I A I 5} F L\right)-A B S\left(L I_{I A I} F L\right)\right)}{4} \\
\text { PERIndex }= & \frac{\text { PERIndexNF }+ \text { PERIndexFR }+ \text { PERIndexFL }}{3}
\end{aligned}
$$

Received December 8, 2011 Revision received August 28, 2012 Accepted October 16, 2012 